\title{
D-dimer in the diagnosis of periprosthetic joint infection: a systematic review and meta-analysis
}

\author{
Guangxu Lu ${ }^{1,2,3}$, Tong Li ${ }^{2}$, Haoqi Ye ${ }^{1}$, Shujin Liu ${ }^{1,2}$, Peng Zhang ${ }^{2}$ and Wenliang Wang ${ }^{2^{*}}$ (D)
}

\begin{abstract}
Background: D-dimer, a coagulation-related indicator, has recently been used as a tool for the diagnosis of periprosthetic joint infection (PJI), but its reliability is uncertain. The purpose of this systematic review and metaanalysis was to explore the accuracy of D-dimer in the diagnosis of PJI after joint arthroplasty.
\end{abstract}

Methods: We systematically searched the MEDLINE, EMBASE, and Cochrane databases for relevant literature about D-dimer in the diagnosis of PJI. QUADAS-2 was used to assess the risk of bias and clinical applicability of each included study. We used the bivariate meta-analysis framework to pool the sensitivity, specificity, positive likelihood ratio (PLR), negative likelihood ratio (NLR), diagnostic odds ratio (DOR), and area under the SROC curve (AUC). Univariate meta-regression and subgroup analyses were performed to explore the sources of heterogeneity.

Results: We included 8 eligible studies. The pooled diagnostic sensitivity and specificity were $0.82(95 \% \mathrm{Cl}$, $0.70-0.89)$ and $0.70(95 \% \mathrm{Cl}, 0.55-0.82)$, respectively. The pooled PLR, NLR, and DOR were $2.7(95 \% \mathrm{Cl}, 1.7-4.4)$, $0.26(95 \% \mathrm{Cl}, 0.15-0.46)$, and 10 (95\% Cl, 4-25), respectively. The AUC was 0.83 (95\% Cl, 0.8-0.86). Serum Ddimer might have higher diagnostic accuracy than plasma D-dimer for PJl (pooled sensitivity: 0.88 vs 0.67; pooled specificity: 0.76 vs 0.61 ).

Conclusions: D-dimer has limited performance for the diagnosis of PJl.

Keywords: Periprosthetic joint infection, D-dimer, Arthroplasty, Diagnosis, Meta-analysis

\section{Introduction}

Periprosthetic joint infection (PJI) is a rare and devastating complication that affects $0.7-2.4 \%$ of patients after hip or knee arthroplasty [1-3]. PJI not only affects the quality of life of infected patients but also increases the risk of death [4].

Because the typical clinical manifestations of patients with PJI may not appear and pain can be caused by other diseases, PJI is difficult to diagnose. The Musculoskeletal Infection Society (MSIS) formulated diagnostic

\footnotetext{
*Correspondence: 1214789714@qq.com

${ }^{2}$ Department of Orthopedics, Characteristic Medical Center of PAP, Tianjin 300162, China

Full list of author information is available at the end of the article
}

criteria for PJI and tried to reduce the incidence rate of this dreaded complication [5, 6]. In 2018, the International Consensus Meeting (ICM) modified the criteria and added D-dimer and alpha-defensin into the new definition of PJI for the knee and hip joint [7] (Table 1).

$\mathrm{D}$-dimer is a specific degradation product of fibrin monomer that is crosslinked by activating factor XIII and then hydrolyzed by fibrinolytic enzyme [8]. It is a specific marker of the fibrinolysis process and mainly reflects the function of fibrinolysis [8]. A study suggested that $\mathrm{D}$-dimer could be used to determine prognosis in systemic sepsis [9]. D-dimer levels continue to rise due to the host's inflammatory response to infection in sepsis [9]. 
Table 1 MSIS criteria for diagnosis of PJI (modified by ICM in 2018) [7]

\begin{tabular}{|c|c|c|c|c|}
\hline \multirow[t]{2}{*}{ Major criteria } & \multicolumn{4}{|c|}{ 1. Two positive periprosthetic cultures with phenotypically identical organisms } \\
\hline & \multicolumn{4}{|c|}{ 2. A sinus tract communicating with the joint } \\
\hline \multirow[t]{12}{*}{ Minor criteria } & \multicolumn{2}{|c|}{ 3. Preoperative diagnosis } & Score & Decision \\
\hline & \multirow[t]{2}{*}{ Serum } & 1).CRP (> $1 \mathrm{mg} / \mathrm{dL}$ ) OR D-dimer (> $850 \mathrm{ng} / \mathrm{mL}$ ) & 2 & \multirow{6}{*}{$\begin{array}{l}\geq 6: \text { Infected } \\
\text { 2-5: Possibly infected } \\
0-1: \text { Not infected }\end{array}$} \\
\hline & & 2). $\operatorname{ESR}(>30 \mathrm{~mm} / \mathrm{h})$ & 1 & \\
\hline & \multirow[t]{4}{*}{ Synovial } & 1). Synovial WBC count (> 3000 cells/uL) or LE + & 3 & \\
\hline & & 2). Alpha-defensin (signal-to cut-off ratio $>1$ ) & 3 & \\
\hline & & 3). Synovial PMN (\%) (> 80\%) & 2 & \\
\hline & & 4). Synovial CRP (> 6.9 mg/L) & 1 & \\
\hline & \multicolumn{2}{|c|}{ 4. Intraoperative diagnosis. } & Score & \multirow{5}{*}{$\begin{array}{l}\geq 6: \text { Infected } \\
\text { 4-5: Inconclusive } \\
\leq 3: \text { Not infected }\end{array}$} \\
\hline & \multicolumn{2}{|c|}{ 1). Preoperative score } & - & \\
\hline & \multicolumn{2}{|c|}{ 2). Histology } & 3 & \\
\hline & \multicolumn{2}{|c|}{ 3). Purulence } & 3 & \\
\hline & \multicolumn{2}{|c|}{ 4). Single culture } & 2 & \\
\hline
\end{tabular}

$P J l$ is present when 1 of the major criteria is met

CRP C-reactive protein, ESR erythrocyte sedimentation rate, WBC white blood cell, and PMN\% polymorphonuclear neutrophil percentage

Currently, some studies have examined the diagnostic value of D-dimer for PJI, but diagnostic accuracy varies in different studies. Therefore, the purpose of this systematic review and meta-analysis was to evaluate the diagnostic accuracy of D-dimer for PJI.

\section{Materials and methods}

This systematic review and meta-analysis strictly followed the Preferred Reporting Items for Systematic Reviews and Meta-Analyses (PRISMA) guidelines [10] (Fig. 1).

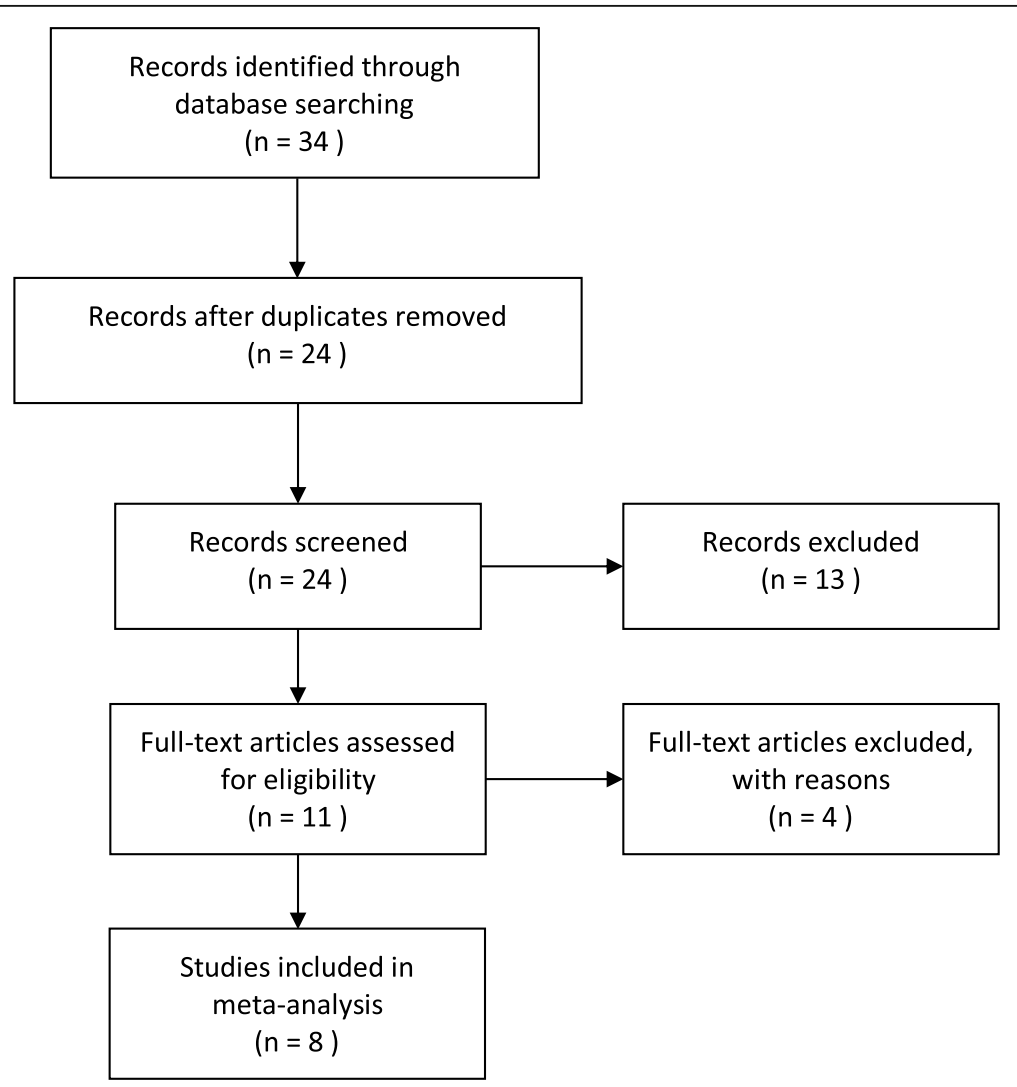

Fig. 1 PRISMA flow diagram 


\section{Search strategy}

We systematically searched all literature about D-dimer in the diagnosis of PJI in MEDLINE, Embase, and the Cochrane Library (from the inception of each database until November 2019), without language restrictions. The search strategies are shown in Table 2.

\section{Eligibility criteria}

We included all studies that reported the accuracy of Ddimer in the diagnosis of PJI after hip or knee arthroplasty and used the MISS or modified MISS criteria. Studies lacking sensitivity and specificity values and those that had duplicated data were excluded.

Two authors independently scanned the titles, abstracts, and full texts sequentially and screened the literature based on the eligibility criteria. The third author settled any disagreements that arose.

\section{Data extraction}

Two authors independently classified all studies and extracted data using standardized scales. We extracted all baseline data (author name, publication year, country, average age, sex distribution, BMI, joint type, patient exclusion criteria, diagnostic criteria, etc.) and outcome indicators (sensitivity, specificity, PLR, NLR, DOR, AUC, etc.). The third author resolved any disagreements that arose.

\section{Quality evaluation}

The quality of each included study was evaluated using the QUADAS-2 tool [11], which mainly includes four

Table 2 Search strategy on MEDLINE, Embase, and Cochrane

\begin{tabular}{l}
\hline PubMed and Cochrane \\
\#1 (((()((()((joint arthroplasty) OR joint replacement) OR knee \\
replacement) OR hip replacement) OR hip arthroplasty) OR knee \\
arthroplasty) OR Arthroplasty, Replacement, Knee) OR Arthroplasty, \\
Replacement) OR Arthroplasty, Replacement, Shoulder) OR Arthroplasty, \\
Replacement, Hip) OR shoulder replacement) OR shoulder arthroplasty \\
\#2 ((periprosthetic infection) OR prosthetic joint infection) OR \\
periprosthetic joint infection \\
\#3 ((diagnostic test) OR test) OR diagnosis \\
\#4 (((((((fibrin fragment D) or D-dimer fibrin) or D-dimer fragments) or fi- \\
brin fragment D1 dimer) or fibrin fragment DD) or D-dimer) or fibrin \\
fragment D-dimer) \\
\#1 and \#2 and \#3 and \#4 \\
EMBASE \\
\#1 'joint arthroplasty' OR 'joint replacement' OR 'knee replacement' OR \\
'hip replacement' OR 'hip arthroplasty' OR 'knee arthroplasty' OR \\
'replacement arthroplasty' OR 'shoulder replacement' OR 'shoulder \\
arthroplasty' \\
\#2 'diagnostic test' OR 'diagnosis' OR test \\
\#3 'periprosthetic infection' OR 'prosthetic joint infection' OR \\
'periprosthetic joint infection' \\
\#4 'fibrin fragment d'/expr OR 'fibrin fragment d' OR 'd-dimer fibrin' OR \\
'd-dimer fragments' OR 'fibrin fragment d1 dimer' OR 'fibrin fragment \\
dd' OR 'd dimer'/exp OR 'd dimer' OR 'fibrin fragment d-dimer' \\
\#1 and \#2 and \#3 and \#4 \\
\hline
\end{tabular}

parts: patient selection, indicator testing, reference standard, and flow and timing. The first three parts are also needed to evaluate clinical practicability. According to the answers ("yes," "no," or "uncertain") to the relevant landmark questions included in each part, the risk of bias level was determined as "low," "high," or "uncertain." Two authors independently evaluated the quality, and the third author decided the final result in the event of any divergences.

\section{Statistical analysis}

We used the bivariate meta-analysis framework to pool the sensitivity, specificity, PLR, NLR, DOR, and AUC by using the "Midas" command [12]. Compared with the traditional summary ROC curve, the bivariate model is a development and expansion [13]. The joint modeling of sensitivity and specificity is used as the starting point for the analysis, and a random effects model is used [13]. Thus, the diagnostic accuracy may be more reliable with this method [14]. The $I^{2}$ statistic was used to estimate the heterogeneity among studies. The value of $I^{2}$ is between 0 and $100 \%$. An $I^{2}$ value of $<50 \%$ indicates low heterogeneity, while an $I^{2}$ value of $>50 \%$ indicates high heterogeneity.

When there was high heterogeneity, we evaluated the threshold effect through the Spearman correlation coefficient of the logarithm of sensitivity and 1-specificity. When the $P$ value was $<0.05$, the threshold effect was considered significant. At the same time, we used univariate meta-regression to find the potential sources of heterogeneity. Then, we conducted a subgroup analysis to further investigate the source of heterogeneity. A test for publication bias (Deeks' funnel plot) was also used to analyze the sources of heterogeneity. When the $P$ value was $<0.05$, the tests for publication bias were considered statistically significant [15].

Stata 14.0 software and Meta-DiSc 1.4 were used for data analysis.

\section{Result}

After a systematic search in the above databases, 34 studies were initially selected, and finally, 8 studies [16-23] were included according to the inclusion and exclusion criteria (Table 3). The 8 included studies were conducted in 2 countries (China and the USA) and included 1587 patients, involving 514 knee joints, 822 hip joints, and 50 extra-articular infections. A total of 457 patients were diagnosed with PJI, and the rate ranged from 17 to $45 \%$. The average age of all the patients in the studies ranged from 61.5 to 68.9 years, with $33-53 \%$ males. All 8 studies were published in the last 3 years, and there was no patient overlap in these studies. 
Table $\mathbf{3}$ Characteristics of included studies

\begin{tabular}{|c|c|c|c|c|c|c|c|c|c|c|c|}
\hline Study & Year & Country & Study design & $\begin{array}{l}\text { No. of } \\
\text { patients }^{\mathrm{a}}\end{array}$ & $\begin{array}{l}\text { Mean } \\
\text { age }\end{array}$ & Mal/female & Site of arthroplasty & $\begin{array}{l}\text { Exclusion } \\
\text { criteria }\end{array}$ & $\begin{array}{l}\text { Reference } \\
\text { standard }\end{array}$ & Cut-off & Sample \\
\hline Shahi et al. [20] & 2017 & USA & Prospective & $57 / 245^{b}$ & 61.5 & $129 / 116$ & Knee (98) and hip (97) & $A$ & MSIS & $850 \mathrm{ng} / \mathrm{ml}$ & Serum \\
\hline Fu et al. [22] & 2019 & China & Prospective & $15 / 45$ & 65.8 & $12 / 33$ & Knee (40) and hip (5) & B & MSIS & $850 \mathrm{ng} / \mathrm{ml}$ & Plasma \\
\hline Li et al. [19] & 2019 & China & Retrospective & $95 / 565$ & 61.7 & $248 / 317$ & $\begin{array}{l}\text { Knee (153) and hip } \\
(412)\end{array}$ & $A$ & ICM & $1250 \mathrm{ng} / \mathrm{ml}$ & Plasma \\
\hline Xu et al. [18] & 2019 & China & Retrospective & $129 / 318$ & NA & NA & Knee (63) and hip (23) & A & MSIS & $1020 \mathrm{ng} / \mathrm{ml}$ & Plasma \\
\hline Huang et al. [21] & 2019 & China & Retrospective & $31 / 101$ & 66.4 & NA & Hip (101) & B & MSIS & $850 \mathrm{ng} / \mathrm{ml}$ & Serum \\
\hline Qin et al. [16] & 2020 & China & Prospective & $55 / 122$ & 65.2 & $53 / 69$ & Knee (44) and hip (78) & B & MSIS & $1170 \mathrm{ng} / \mathrm{ml}$ & Serum \\
\hline Xiong et al. [17] & 2019 & China & Prospective & $26 / 80$ & 62.3 & $32 / 48$ & Knee (47) and hip (33) & B & MSIS & $760 \mathrm{ng} / \mathrm{ml}$ & Serum \\
\hline Pannu et al. [23] & 2020 & USA & Retrospective & $49 / 111$ & 68.9 & $49 / 111$ & Knee (69) and hip (42) & A & ICM & $850 \mathrm{ng} / \mathrm{ml}$ & serum \\
\hline
\end{tabular}

$A$ does not exclude patients with rheumatoid arthritis, autoimmune diseases, tumors, smoking and obesity; $B$ exclude patients with rheumatoid arthritis, autoimmune diseases, tumors, smoking, and obesity

NA not available

${ }^{a}$ The values are given as the number of patients with an infection/total number of patients in study

${ }^{b_{50}}$ of 245 patients were extra-articular infection

Four studies [16, 17, 20, 22] were prospective studies, and the other 4 studies $[18,19,21,23]$ were retrospective studies. In terms of the diagnostic threshold, 4 studies [20-23] used $850 \mathrm{~g} / \mathrm{L}$, which was recommended by the ICM (2018) as the diagnostic threshold of D-dimer. Pannu et al. [23] also used $2300 \mathrm{ng} / \mathrm{ml}$ as the cut-off in their study. The remaining 4 studies [16-19] used 1250 $\mathrm{ng} / \mathrm{L}, 1020 \mathrm{ng} / \mathrm{L}, 1170 \mathrm{ng} / \mathrm{ml}$, and $760 \mathrm{ng} / \mathrm{ml}$ as the diagnostic thresholds. Four studies [21-23] determined the diagnostic threshold in advance, and the remaining studies [16-20] obtained the diagnostic threshold from the ROC curve. Three studies $[18,19,22,23]$, all from China, used plasma samples for the quantification of Ddimer, and 5 studies $[16,17,20,21,23]$ used serum samples. Four studies $[16,17,21,22]$ excluded patients with rheumatoid arthritis, autoimmune diseases, tumors, smoker status, or obesity and the remaining 4 studies [18-20, 23] did not.

\section{Quality assessment}

According to the QUADAS-2 tool, we evaluated the quality of all included studies (Table 4 and Fig. 2). The risk of bias in reference standards and flow and timing was low in all studies. Six studies [16-18, 20-22] were at high risk of bias for patient selection because of inappropriate discharge standards and case-control trials. Because retrospective studies and thresholds were not set in advance in 7 studies [16-21, 23], the bias of the index test was high. All studies scored between 6 and 9 (the total score is 10 points).

Table 4 QUADAS-2 evaluation

\begin{tabular}{|c|c|c|c|c|c|c|c|c|c|c|c|c|c|c|c|c|c|}
\hline \multirow[t]{2}{*}{ Study } & \multicolumn{17}{|c|}{ QUADAS Score } \\
\hline & 1 & 2 & 3 & Bias & Appl. & 4 & 5 & Bias & Appl. & 6 & 7 & Bias & Appl. & 8 & 9 & 10 & Bias \\
\hline Shahi et al. [20] & NC & 0 & 1 & High & Low & 1 & 0 & High & Low & 1 & 1 & Low & Low & 1 & 1 & 1 & $\overline{\text { Low }}$ \\
\hline Fu et al. [22] & NC & 0 & 0 & High & Low & 1 & 1 & Low & Low & 1 & 1 & Low & Low & 1 & 1 & 1 & Low \\
\hline Li et al. [19] & NC & 1 & 1 & Low & Low & 0 & 0 & High & Low & 1 & 1 & Low & Low & 1 & 1 & 1 & Low \\
\hline Xu et al. [18] & NC & 0 & 1 & High & Low & 0 & 0 & High & Low & 1 & 1 & Low & Low & 1 & 1 & 1 & Low \\
\hline Huang et al. [21] & NC & 0 & 0 & High & Low & 0 & 1 & High & Low & 1 & 1 & Low & Low & 1 & 1 & 1 & Low \\
\hline Qin et al. [16] & NC & 1 & 0 & High & Low & 1 & 0 & High & Low & 1 & 1 & Low & Low & 1 & 1 & 1 & Low \\
\hline Xiong et al. [17] & NC & 1 & 0 & High & Low & 1 & 0 & High & Low & 1 & 1 & Low & Low & 1 & 1 & 1 & Low \\
\hline Pannu et al. [23] & 1 & 1 & 1 & Low & Low & 0 & 1 & High & Low & 1 & 1 & Low & Low & 1 & 1 & 1 & Low \\
\hline
\end{tabular}

The numbers in the top row correspond to the following questions: Domain 1: Patient selection. Numbers correspond with the following questions: (1) Was a consecutive or random sample of patients enrolled? (2) Was a case-control design avoided? (3) Did the study avoid inappropriate exclusions? Domain 2: Index test. Numbers correspond with the following questions: (4) Were the index test results interpreted without knowledge of the results of the reference standard? (5) If a threshold was used, was it pre-specified? Domain 3: Reference test. Numbers correspond with the following questions: (6) Is the reference standard likely to correctly classify the target condition? (7) Were the reference standard results interpreted without knowledge of the results of the index test? Domain 4: Flow and timing. Numbers correspond with the following questions: (8) Was there an appropriate interval between index test(s) and reference standard? (9) Did all patients receive a reference standard? (10) Did patients receive the same reference standard? (11) Were all patients included in the analysis?

*Number 1 indicates "yes," and 0 indicates "no"; Bias risk: of bias; Appl.: concerns regarding applicability; NC: not clear 


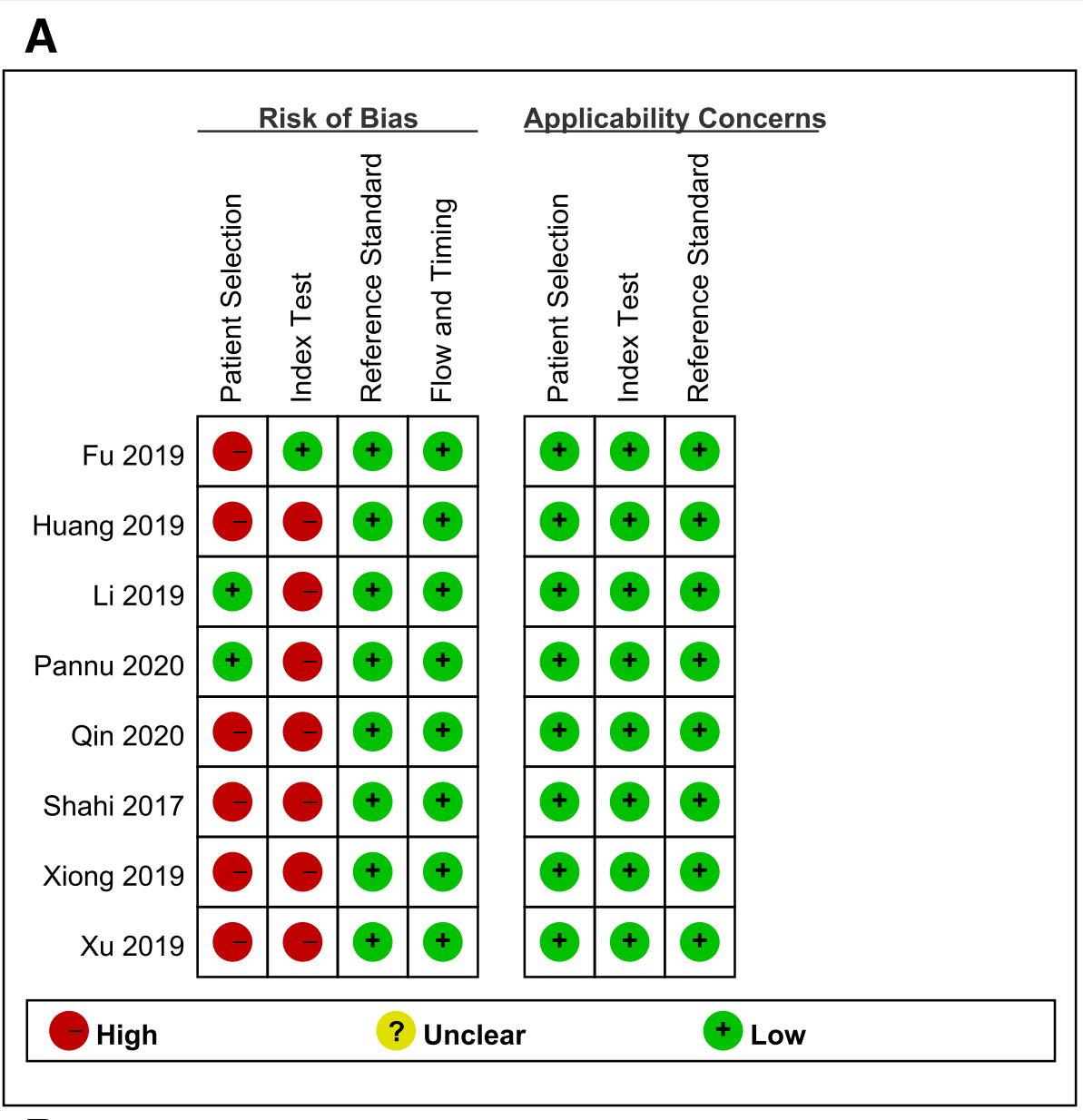

B

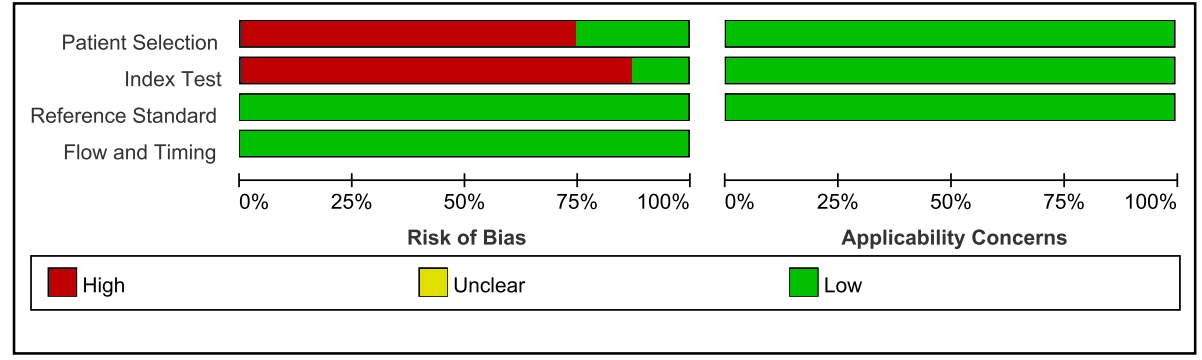

Fig. 2 Risk of bias and applicability concerns summary. a Risk of bias and applicability concerns graph (b)

\section{Diagnostic value}

The pooled diagnostic sensitivity and specificity were 0.82 (95\% CI, 0.70-0.89) and 0.70 (95\% CI, 0.55-0.82), respectively (Fig. 3); however, the heterogeneity between studies was obvious, with $I^{2}$ values of $83.19 \%$ (95\% CI, 71.75-94.64\%) and 94.17\% (95\% CI, 91.23-97.11\%). The pooled PLR, NLR, and DOR were 2.7 (95\% CI, 1.7-5.4), 0.26 (95\% CI, 0.15-0.46), and 10 (95\% CI, 4-25), respectively (Fig. 3). The AUC was 0.83 (95\% CI, 0.8-0.86) (Fig. 4). The Spearman correlation coefficient was -
$0.071(P=0.867)$. The heterogeneity might be unrelated to the threshold effects.

\section{Heterogeneity analysis Meta-regression}

We performed univariate meta-regression to search for the potential sources of heterogeneity (Fig. 5). For sensitivity and specificity, the sample differences and racial differences had the most significant impacts on the heterogeneity of the results $(P<0.05)$. Based on these 


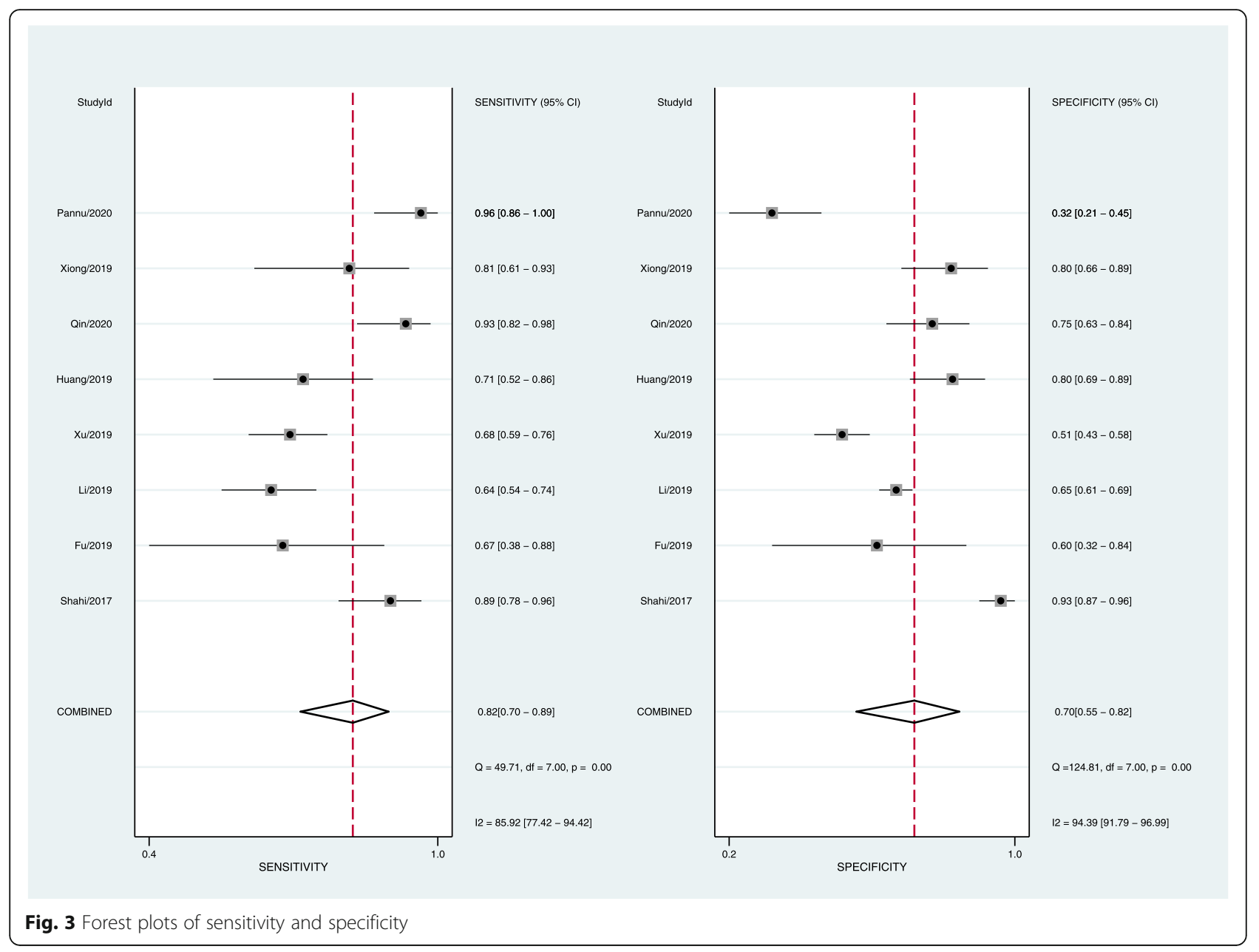

results, we performed subgroup analysis to further explore the source of heterogeneity. When $I^{2}<50 \%$ or $P>0.05$, we considered the heterogeneity to be low in the subgroup.

\section{Subgroup analysis}

In the subgroup of plasma D-dimer [18, 19, 22], the pooled sensitivity and specificity were $0.67(95 \% \mathrm{CI}$ $0.60-0.72$ ) and 0.61 (95\% CI 0.57-0.65); in the subgroup of serum D-dimer $[16,17,20,21,23]$, the pooled sensitivity and specificity were 0.88 (95\% CI $0.83-0.92)$ and 0.76 (95\% CI 0.71-0.80). In the subgroup of East Asian races $[16-19,21,22]$, the pooled sensitivity and specificity were 0.72 (95\% CI $0.67-0.77)$ and 0.65 (95\% CI 0.61-0.68); in the subgroup of Caucasian and African American races [20, 23], the pooled sensitivity and specificity were 0.92 (95\% CI $0.86-0.97)$ and 0.74 (95\% CI $0.67-0.80$ ), respectively (Table 5 ).

\section{Publication bias}

The Deeks' funnel plot asymmetry test of DOR did not show significant asymmetry $(P=0.34)$, indicating that publication bias might not exist (Fig. 6).

\section{Discussion}

The diagnosis of PJI after arthroplasty is a complicated problem for every orthopedist. With early diagnosis, patients can undergo debridement or conservative treatment to treat PJI and avoid 1 or 2 stage revision. Therefore, the quick and accurate diagnosis of PJI is critical. Many potential blood and synovial fluid biomarkers for the diagnosis of PJI have been evaluated, but the clinical gold standard for the diagnosis of the disease has still not been found. Therefore, it is necessary and meaningful to develop a new and accurate diagnostic method for PJI.

D-dimer is familiar to medical workers and has not been valued in the past few decades. It has only been used to screen venous thromboembolism [24, 25]. Recently, some studies showed that D-dimer was associated with inflammation and might be elevated in infected patients [26, 27]. Rodelo et al. found that higher levels of D-dimer were associated with increased 28-day mortality in septic patients [9]. In addition, D-dimer is recommended as a critical diagnostic indicator for infectious diseases such as endocarditis and mycoplasma 


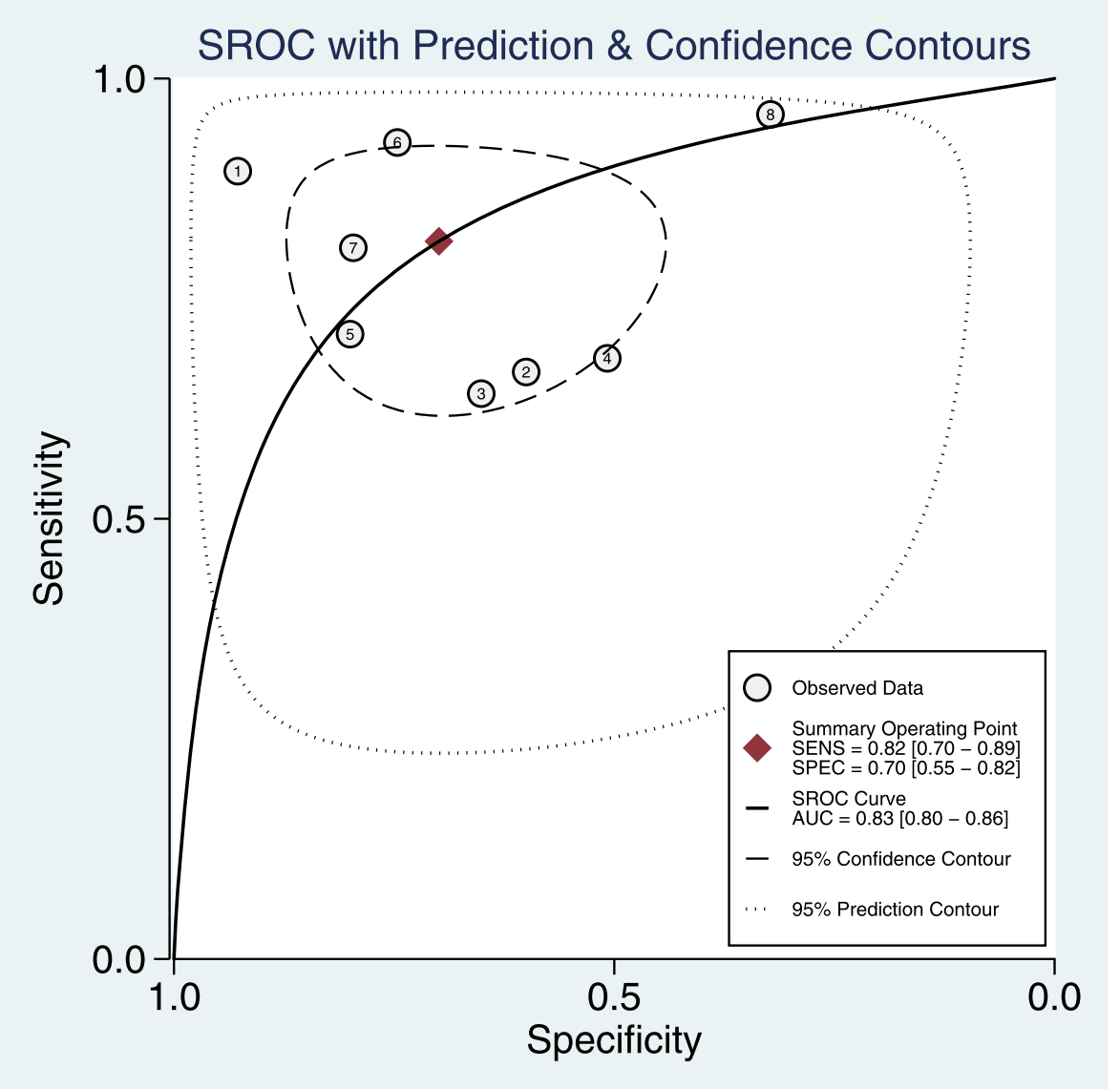

Fig. 4 SROC curve of sequencing-based diagnosis performance

pneumonia [28, 29]. Subsequently, D-dimer levels attracted the attention of plastic surgeons.

Shahi et al. [20] reported in his study that serum Ddimer has high diagnostic value for PJI in lower limbs, with a sensitivity and specificity of $89 \%$ and $93 \%$, respectively, which preluded the diagnosis of PJI by Ddimer. Parvizi et al. [30] believe that the diagnosis of PJI, such as ankylosing spondylitis, rheumatoid arthritis, and endocarditis, should depend on a combination of various diagnoses, so they added D-dimer and redefined the diagnosis of the PJI standard. The new diagnostic criteria were validated in 222 PJI patients and 200 sterile patients. They found that the sensitivity and specificity of the new diagnostic criteria were $97.7 \%$ and $99.5 \%$, respectively, while the sensitivities of the MSIS and ICM diagnostic criteria were only $86.9 \%$ and $79.3 \%$, and their specificities were both $99.5 \%$. The ICM passed this diagnostic criterion in 2018, but the pass rate was only $68 \%$. Since 2019, an increasing number of articles about Ddimer in the diagnosis of PJI have been reported, and its diagnostic value is suspected.
This is the first systematic review and meta-analysis about the utility of D-dimer for the diagnosis of PJI. We found that D-dimer has limited performance for the diagnosis of PJI, with a pooled sensitivity and specificity of 0.82 and 0.70 , respectively, and had a poorer diagnostic value than that of CPR and ESR reported by Carli AV et al. [31]. In this systematic review, the pooled sensitivity and specificity of CRP were 0.85 and 0.81 , respectively, and the pooled sensitivity and specificity of ESR were 0.82 and 0.79 . The results of the subgroup analysis showed that serum D-dimer might have a higher diagnostic accuracy than plasma D-dimer for PJI (the pooled sensitivity was 0.88 vs 0.67 , and the pooled specificity was 0.76 vs 0.61 ), and D-dimer had better accuracy in subgroups with Caucasian and African American races than in subgroups with East Asian races (the pooled sensitivity was 0.92 vs 0.72 , and the pooled specificity was 0.74 vs 0.65 ).

One possible reason for the variance in the subgroup results was that the samples for the quantification of Ddimer were different: serum vs plasma. Serum is the 


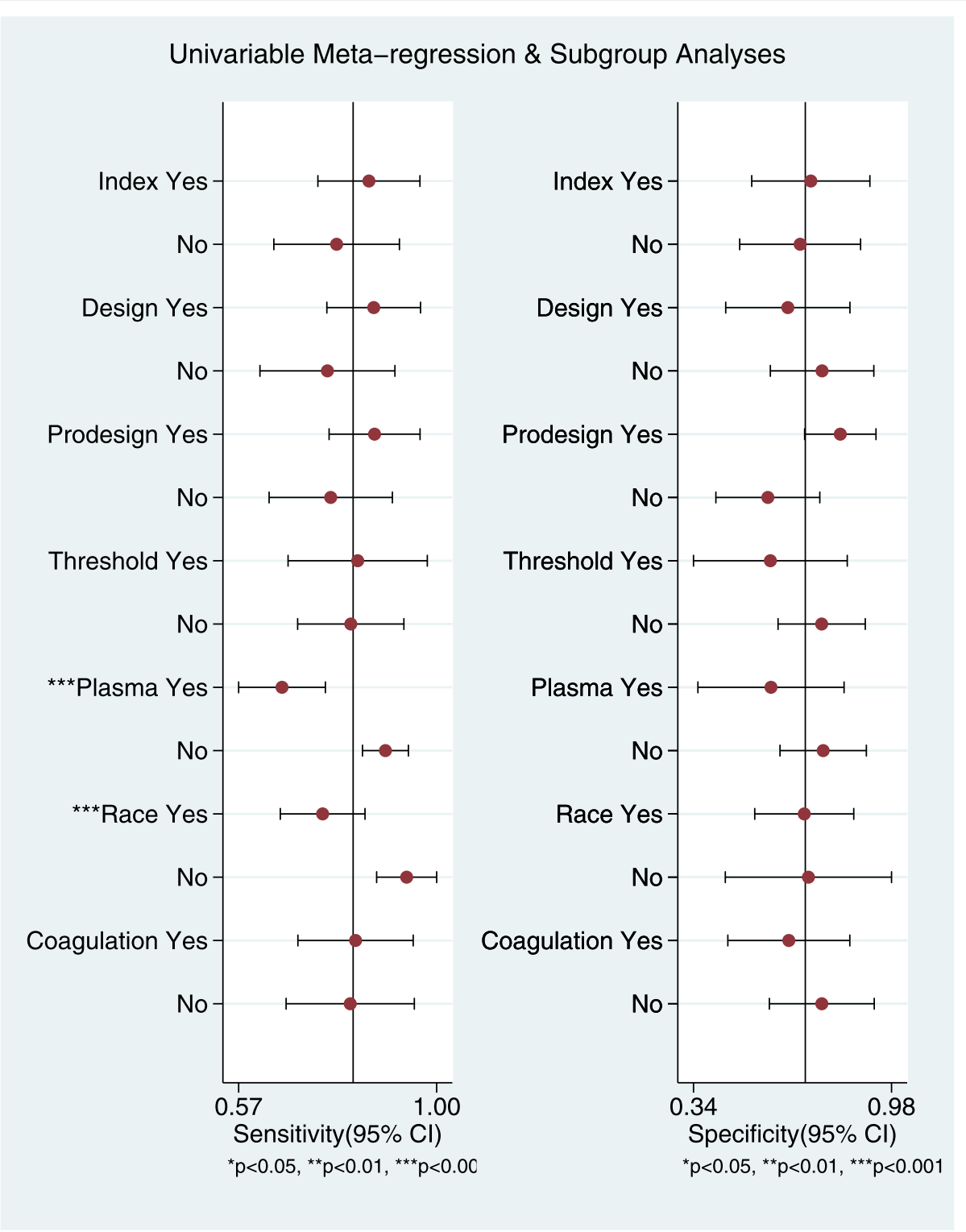

Fig. 5 Univariable meta-regression

liquid part of blood after coagulation, while plasma is the liquid part of the blood where coagulation has been prevented. Their density is similar, but their composition is different. The main difference is that there are more fibrinogen and coagulation proteins in plasma [32].
Boisclair et al. [33] reported that there was a very high correlation between plasma and serum D-dimer levels $(r=0.931, P<0.01)$, but the diagnostic sensitivity was not consistent. The study reported that the sensitivities of plasma D-dimer for DIC, DVT, and MI

Table 5 Subgroup analysis

\begin{tabular}{llllll}
\hline Subgroup & Number of studies & Pooled sensitivity $(95 \% \mathrm{Cl})$ & Pooled specificity $(95 \% \mathrm{Cl})$ & $\boldsymbol{P}$ & $\boldsymbol{I}^{\mathbf{2}}$ \\
\hline A & 3 & $0.67(0.60-0.72)$ & $0.61(0.57-0.65)$ & $0.82 / 0.003$ & $0 / 82.6 \%$ \\
B & 5 & $0.88(0.83-0.92)$ & $0.76(0.71-0.80)$ & $0.0001 / 0.0001$ & $68.7 \% / 95.1 \%$ \\
C & 6 & $0.72(0.67-0.77)$ & $0.65(0.61-0.68)$ & $0.001 / 0.0001$ & $74.9 \% / 84.5 \%$ \\
D & 2 & $0.92(0.86-0.97)$ & $0.74(0.67-0.80)$ & $0.20 / 0.0001$ & $39.5 \% / 98.7 \%$
\end{tabular}

A plasma D-dimer, $B$ serum D-dimer, C East Asian race, $D$ Caucasian and African American race 


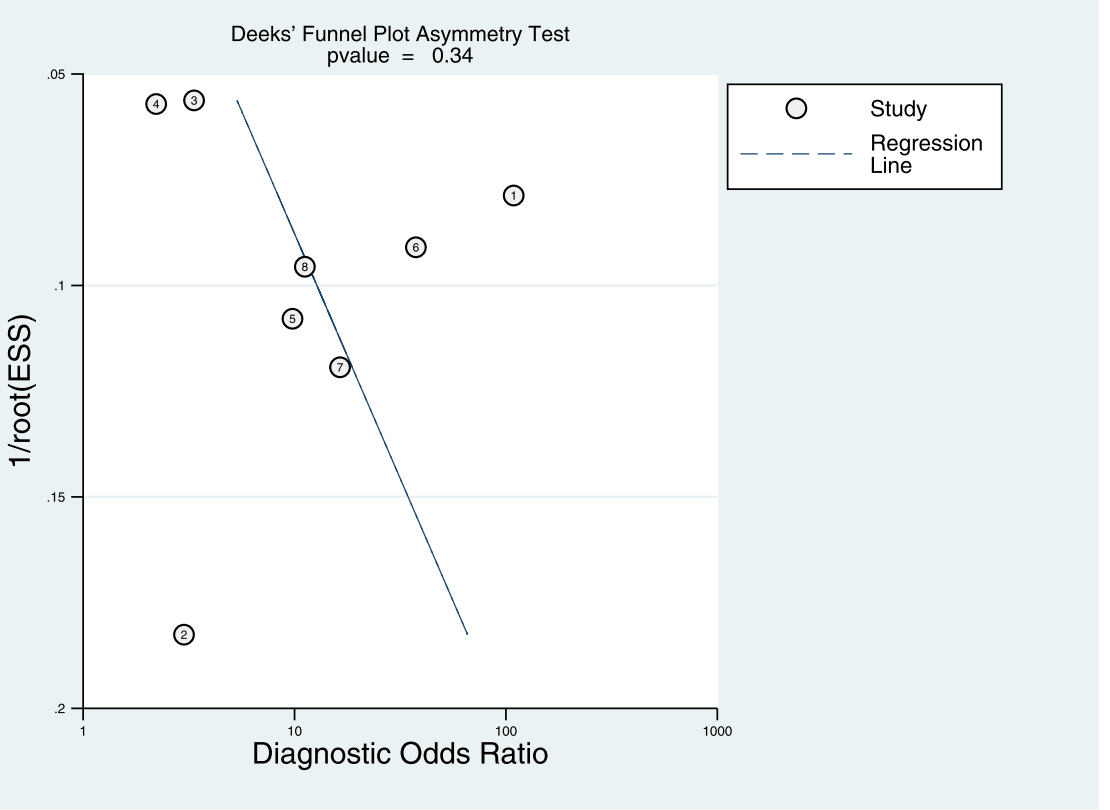

Fig. 6 The Deeks' funnel plot of the pooled DOR

were $100 \%, 90.4 \%$, and $60 \%$, respectively, but the sensitivities of serum D-dimer were $100 \%, 94.1 \%$, and $22.2 \%$. The different sensitivities of plasma and serum might be due to the more significant uncertainty in assigning a cut-off for elevated levels of serum Ddimer. The D-dimer assay was operating at its lower detection limit when used to measure non-elevated levels in serum [33]. However, whether different sensitivities between plasma and serum exist in PJI is not supported by relevant literature.

Another possible reason was that the level of D-dimer is easily affected by other diseases. Busso et al. [34] reported that the inflammatory synovium secretes a large amount of fibrin in patients with rheumatoid arthritis, and the degradation of this protein subsequently leads to an increase in the level of D-dimer in serum and synovial fluid. In addition, thrombosis [35], malignancies, autoimmune diseases, pregnancy, and heart and brain vascular diseases might affect the determination of $\mathrm{D}$ dimer levels in the blood [36, 37]. Li et al. [19] found that the diagnostic accuracy of D-dimer was poor in the subgroups containing these diseases in their study.

In addition, racial differences may affect the diagnostic accuracy of D-dimer for PJI. Shahi and Pannu's studies $[20,23]$ were conducted in the USA, and the population may be predominantly Caucasian and African-American. In the six other studies reported by Chinese scholars, the patients were predominantly of the East Asian race. The studies found that D-dimer levels varied between races, such as between African American and Caucasian patients $[38,39]$. We suspect that there are also differences in D-dimer levels between the East Asian population and the other races, which will affect the result. However, there are no studies to support this view.

Synovial fluid viscosity tests and several other plasma biomarkers have been reported to diagnose PJI. The synovial fluid viscosity level was significantly lower in patients with PJI than in patients with aseptic failure, with a sensitivity of 0.99 and a specificity of 0.67 [22]. Both plasma fibrinogen and fibrin degradation product (FDP) are coagulation-related indicators. When the threshold for plasma fibrinogen was $4.01 \mathrm{~g} / \mathrm{L}$, the sensitivity and specificity values were 0.763 and 0.862 [19], respectively. FDP has low sensitivity and specificity, with values of $65.12 \%$ and $60.33 \%$, respectively [18].

Our meta-analysis has some strengths and potential limitations. The cases included all involved hip and knee joints. In addition, all studies used MSIS standards [5] or modified MSIS standards [6]. Therefore, the classification bias was minimized. The most important factor was that all D-dimer tests were taken before surgery, excluding the interference of a sharp increase in serum Ddimer levels after surgery [40].

The limitations of our meta-analysis included variability in race, age range, sex ratio, and sample size. In addition, none of the studies considered whether patients used antibiotics before admission. Shahi et al. [20] reported that premature antibiotic treatment could affect the results of D-dimer in the blood. Another limitation of our study is that MSIS standards or modified MSIS 
standards lack the sensitivity to detect chronic and lowgrade PJI; patients with "positive" D-dimer results might be classified as uninfected [41]. Additionally, most studies did not provide information about the measurement of D-dimer. D-dimer assays can be categorized into three types [42]: ELISA, immunoturbidimetric automated assay, and latex-based immunoassays. ELISA is more sensitive than immunoturbidimetric automated assays and latex-based immunoassays [42]. In addition, some studies excluded patients with tumors, rheumatoid arthritis, autoimmune diseases, a history of smoking, and obesity. However, the proportion of such patients in joint replacement is still high. The exclusion of these patients will interfere with the accuracy of D-dimer in the diagnosis of PJI. Finally, the diagnostic thresholds in some studies were not determined in advance, and the threshold values were not unified in this meta-analysis.

\section{Conclusion}

D-dimer, a coagulation-related indicator, is inexpensive and easy to measure but has limited performance for the diagnosis of PJI, and the pooled sensitivity and specificity were poorer than those of traditional inflammatory markers such as CRP and ESR. Based on our findings, we suggest using serum samples for the quantification of D-dimer. Additionally, the diagnostic accuracy may be better in Caucasian and African American patients.

\begin{abstract}
Abbreviations
PJl: Periprosthetic joint infection; PLR: Positive likelihood ratio; NLR: Negative likelihood ratio; DOR: Diagnostic odds ratio; AUC: The area under the SROC curve; MSIS: Musculoskeletal Infection Society; ICM: International Consensus Meeting; CRP: C-reactive protein; ESR: Erythrocyte sedimentation rate; WBC: White blood cell; PMN\%: Polymorphonuclear neutrophil percentage; PRISMA: Preferred Reporting Items for Systematic Reviews and Meta-Analyses
\end{abstract}

\section{Acknowledgements}

Not applicable

\begin{abstract}
Authors' contributions
Guangxu Lu, first author, searched the database, performed the metaanalysis, and drafted the manuscript. Tong Li, co-first author, participated in the design of the study, searched the database, and performed the metaanalysis. Haogi Ye participated in the design of the study, searched the database, and performed the meta-analysis. Shujing Liu participated in the design of the study, searched the database, and performed the meta-analysis. Peng Zhang participated in the design of the study, searched the database, and performed the meta-analysis. Wenliang Wang participated in the design of the study and helped to draft the manuscript. The authors read and approved the final manuscript.
\end{abstract}

\section{Funding}

This study was funded by the National Natural Science Foundation of China, China Program (No. 30740089).

\section{Availability of data and materials}

Not applicable

\section{Ethics approval and consent to participate}

Not applicable

\section{Consent for publication}

Not applicable

\section{Competing interests}

No author associated with this paper has disclosed any potential or pertinent conflicts which may be perceived to have impending conflict with this work.

\section{Author details}

'Department of Logistics University of PAP, Tianjin 300309, China. ${ }^{2}$ Department of Orthopedics, Characteristic Medical Center of PAP, Tianjin 300162, China. ${ }^{3}$ Department of Orthopedics, Er Quan Hospital of PAP, Wuxi 214000, China.

Received: 20 March 2020 Accepted: 23 June 2020

Published online: 16 July 2020

\section{References}

1. Kurtz SM, Lau E, Watson H, Schmier JK, Parvizi J. Economic burden of periprosthetic joint infection in the United States. J Arthroplasty. 2012;27(8 Suppl):61-5.e1.

2. Huotari $\mathrm{K}$, Peltola $\mathrm{M}$, Jamsen $\mathrm{E}$. The incidence of late prosthetic joint infections: a registry-based study of 112,708 primary hip and knee replacements. Acta Orthop. 2015;86(3):321-5.

3. Dale H, Fenstad AM, Hallan G, Havelin LI, Furnes O, Overgaard S, et al. Increasing risk of prosthetic joint infection after total hip arthroplasty. Acta Orthop. 2012;83(5):449-58.

4. Berend KR, Lombardi AV Jr, Morris MJ, Bergeson AG, Adams JB, Sneller MA. Two-stage treatment of hip periprosthetic joint infection is associated with a high rate of infection control but high mortality. Clin Orthop Relat Res. 2013;471(2):510-8.

5. Zmistowski B, Della Valle C, Bauer TW, Malizos KN, Alavi A, Bedair H et al. Diagnosis of periprosthetic joint infection. J Orth Res. 2014; 32(Suppl 1):S98-107.

6. Parvizi J, Zmistowski B, Berbari EF, Bauer TW, Springer BD, Della Valle CJ, et al. New definition for periprosthetic joint infection: from the Workgroup of the Musculoskeletal Infection Society. Clin Orthop Relat Res. 2011;469(11): 2992-4.

7. Shohat N, Bauer T, Buttaro M, Budhiparama N, Cashman J, Della Valle CJ, et al. Hip and Knee Section, What is the definition of a Periprosthetic Joint Infection (PJI) of the knee and the hip? Can the same criteria be used for both joints?: Proceedings of International Consensus on Orthopedic Infections. The Journal of arthroplasty. 2019;34(2S):S325-S7.

8. Adam SS, Key NS, Greenberg CS. D-dimer antigen: current concepts and future prospects. Blood. 2009;113(13):2878-87.

9. Rodelo JR, De la Rosa G, Valencia ML, Ospina S, Arango CM, Gomez Cl, et al, D-dimer is a significant prognostic factor in patients with suspected infection and sepsis. Am J Emerg Med. 2012;30(9):1991-9.

10. Moher D, Liberati A, Tetzlaff J, Altman DG. Preferred reporting items for systematic reviews and meta-analyses: the PRISMA statement. J Clin Epidemiol. 2009;62(10):1006-12.

11. Whiting PF, Rutjes AW, Westwood ME, Mallett S, Deeks JJ, Reitsma JB, et al. QUADAS-2: a revised tool for the quality assessment of diagnostic accuracy studies. Ann Intern Med. 2011;155(8):529-36.

12. Dwamena BA. Evidence-based radiology: step 3--diagnostic systematic review and meta-analysis (critical appraisal). Semin Roentgenol. 2009;44(3): 170-9.

13. Dora C, Altwegg M, Gerber C, Bottger EC, Zbinden R. Evaluation of conventional microbiological procedures and molecular genetic techniques for diagnosis of infections in patients with implanted orthopedic devices. J Clin Microbiol. 2008;46(2):824-5.

14. Kriston L, Harter M, Holzel L. Challenges in reporting meta-analyses of diagnostic accuracy studies. Ann Intern Med. 2009;150(6):430.

15. Deeks JJ, Macaskill P, Irwig L. The performance of tests of publication bias and other sample size effects in systematic reviews of diagnostic test accuracy was assessed. J Clin Epidemiol. 2005;58(9):882-93.

16. Qin L, Li F, Gong X, Wang J, Huang W, Hu N. Combined measurement of Ddimer and C-reactive protein levels: highly accurate for diagnosing chronic periprosthetic joint infection. J Arthroplasty. 2020;35(1):229-34.

17. Xiong L, Li S, Dai M. Comparison of D-dimer with CRP and ESR for diagnosis of periprosthetic joint infection. J Orthop Surg Res. 2019;14(1):240.

18. Xu H, Xie J, Huang Q, Lei Y, Zhang S, Pei F. Plasma fibrin degradation product and D-dimer are of limited value for diagnosing periprosthetic joint infection. J Arthroplasty. 2019;34(10):2454-60. 
19. Li R, Shao HY, Hao LB, Yu BZ, Qu PF, Zhou YX, et al. Plasma fibrinogen exhibits better performance than plasma D-dimer in the diagnosis of periprosthetic joint infection: a multicenter retrospective study. J Bone Joint Surg Am. 2019;101(7):613-9.

20. Shahi A, Kheir MM, Tarabichi M, Hosseinzadeh HRS, Tan TL, Parvizi J. Serum D-dimer test is promising for the diagnosis of periprosthetic joint infection and timing of reimplantation. J Bone Joint Surg Am. 2017;99(17):1419-27.

21. Huang J, Zhang Y, Wang Z, Dong Y, Zhao Y, Zheng J, et al. The serum level of D-Dimer is not suitable for distinguishing between prosthetic joint infection and aseptic loosening. J Orthop Surg Res. 2019;14(1):407.

22. Fu J, Ni M, Chai W, Li X, Hao L, Chen J. Synovial fluid viscosity test is promising for the diagnosis of periprosthetic joint infection. J Arthroplasty. 2019;34(6):1197-200

23. Pannu TS, Villa JM, Patel PD, Riesgo AM, Barsoum WK, Higuera CA. The utility of serum D-dimer for the diagnosis of periprosthetic joint infection in revision total hip and knee arthroplasty. The Journal of arthroplasty. 2020: S0883-5403(20)30071-1.

24. Khanbhai M, Hansrani V, Burke J, Ghosh J, McCollum C. The early management of DVT in the North West of England: a nation-wide problem? Thromb Res. 2015;136(1):76-86.

25. Bounameaux $\mathrm{H}$, de Moerloose P, Perrier A, Reber G. Plasma measurement of D-dimer as diagnostic aid in suspected venous thromboembolism: an overview. Thromb Haemost. 1994;71(1):1-6.

26. Schwameis M, Steiner MM, Schoergenhofer C, Lagler H, Buchtele N, JilmaStohlawetz $\mathrm{P}$, et al. D-dimer and histamine in early stage bacteremia: a prospective controlled cohort study. Eur J Intern Med. 2015;26(10):782-6.

27. Ribera T, Monreal L, Armengou L, Rios J, Prades M. Synovial fluid D-dimer concentration in foals with septic joint disease. J Vet Intern Med. 2011;25(5): 1113-7.

28. Turak O, Canpolat U, Ozcan F, Yayla C, Mendi MA, Oksuz F, et al. D-dimer level predicts in-hospital mortality in patients with infective endocarditis: a prospective single-centre study. Thromb Res. 2014;134(3):587-92.

29. Mele N, Turc G. Stroke associated with recent mycoplasma pneumoniae infection: a systematic review of clinical features and presumed pathophysiological mechanisms. Front Neurol. 2018;9:1109.

30. Parvizi J, Tan TL, Goswami K, Higuera C, Della Valle C, Chen AF, et al. The 2018 definition of periprosthetic hip and knee infection: an evidence-based and validated criteria. J Arthroplasty. 2018;33(5):1309-14.e2.

31. Carli AV, Abdelbary H, Ahmadzai N, Cheng W, Shea B, Hutton B, et al. Diagnostic accuracy of serum, synovial, and tissue testing for chronic periprosthetic joint infection after hip and knee replacements: a systematic review. J Bone Joint Surg Am. 2019;101(7):635-49.

32. Lima-Oliveira G, Monneret D, Guerber F, Guidi GC. Sample management for clinical biochemistry assays: are serum and plasma interchangeable specimens? Crit Rev Clin Lab Sci. 2018;55(7):480-500.

33. Boisclair MD, Lane DA, Wilde JT, Ireland H, Preston FE, Ofosu FA. A comparative evaluation of assays for markers of activated coagulation and/ or fibrinolysis: thrombin-antithrombin complex, D-dimer and fibrinogen/ fibrin fragment E antigen. Br J Haematol. 1990;74(4):471-9.

34. Busso N, Hamilton JA. Extravascular coagulation and the plasminogen activator/plasmin system in rheumatoid arthritis. Arthritis Rheum. 2002;46(9): 2268-79.

35. Coleman DM, Wakefield TW. Biomarkers for the diagnosis of deep vein thrombosis. Expert Opin Med Diagn. 2012;6(4):253-7.

36. Olson JD. D-dimer: an overview of hemostasis and fibrinolysis, assays, and clinical applications. Adv Clin Chem. 2015;69:1-46.

37. O'Neal WT, Soliman EZ, Howard G, Howard VJ, Safford MM, Cushman M, et al. Inflammation and hemostasis in atrial fibrillation and coronary heart disease: the REasons for Geographic And Racial Differences in Stroke study. Atherosclerosis. 2015;243(1):192-7.

38. Pieper CF, Rao KM, Currie MS, Harris TB, Cohen HJ. Age, functional status, and racial differences in plasma D-dimer levels in community-dwelling elderly persons. J Gerontol A Biol Sci Med Sci. 2000;55(11):M649-57.

39. Zakai NA, McClure LA, Judd SE, Kissela B, Howard G, Safford M, et al. Ddimer and the risk of stroke and coronary heart disease. The REasons for Geographic and Racial Differences in Stroke (REGARDS) Study. Thromb Haemost. 2017;117(3):618-24

40. Lee YS, Lee YK, Han SB, Nam CH, Parvizi J, Koo KH. Natural progress of Ddimer following total joint arthroplasty: a baseline for the diagnosis of the early postoperative infection. J Orthop Surg Res. 2018;13(1):36.
41. Osmon DR, Berbari EF, Berendt AR, Lew D, Zimmerli W, Steckelberg JM, et al. Diagnosis and management of prosthetic joint infection: clinical practice guidelines by the Infectious Diseases Society of America. Clin Infect Dis. 2013;56(1):e1-e25.

42. Tripodi A. D-dimer testing in laboratory practice. Clin Chem. 2011;57(9): 1256-62.

\section{Publisher's Note}

Springer Nature remains neutral with regard to jurisdictional claims in published maps and institutional affiliations.

\section{Ready to submit your research? Choose BMC and benefit from:}

- fast, convenient online submission

- thorough peer review by experienced researchers in your field

- rapid publication on acceptance

- support for research data, including large and complex data types

- gold Open Access which fosters wider collaboration and increased citations

- maximum visibility for your research: over $100 \mathrm{M}$ website views per year

At BMC, research is always in progress.

Learn more biomedcentral.com/submissions 